\title{
Phonon Squeezed States: Quantum Noise Reduction in Solids
}

\author{
Xuedong $\mathrm{Hu}^{1}$ and Franco Nori ${ }^{2}$ \\ 1 Department of Physics, University of Maryland, College Park, MD 20742 \\ 2 Department of Physics, The University of Michigan, Ann Arbor, Michigan 48109-1120
}

(July 7, 2018)

\begin{abstract}
This article discusses quantum fluctuation properties of a crystal lattice, and in particular, phonon squeezed states. Squeezed states of phonons allow a reduction in the quantum fluctuations of the atomic displacements to below the zero-point quantum noise level of coherent phonon states. Here we discuss our studies of both continuous-wave and impulsive second-order Raman scattering mechanisms. The later approach was used to experimentally suppress (by one part in a million) fluctuations in phonons. We calculate the expectation values and fluctuations of both the atomic displacement and the lattice amplitude operators, as well as the effects of the phonon squeezed states on macroscopically measurable quantities, such as changes in the dielectric constant. These results are compared with recent experiments. Further information, including preprints and animations, are available in http://www-personal.engin.umich.edu/ nori/squeezed.html
\end{abstract}

\section{INTRODUCTION}

Classical phonon optics [i] has succeeded in producing many acoustic analogs of classical optics, such as phonon mirrors, phonon lenses, phonon filters, and even "phonon microscopes" that can generate acoustic pictures with a resolution comparable to that of visible light microscopy. Most phonon optics experiments use heat pulses or superconducting transducers to generate incoherent phonons, which propagate ballistically in the crystal. These ballistic incoherent phonons can then be manipulated by the above-mentioned devices, just like in geometric optics. Phonons can also be excited phase-coherently. For instance, coherent acoustic waves with frequencies of up to $10^{10}$ $\mathrm{Hz}$ can be generated by piezoelectric oscillators. Lasers have also been used to generate coherent acoustic and optical phonons through stimulated Brillouin and Raman scattering experiments. Furthermore, in recent years, it has been possible to track the phases of coherent optical phonons [2] due to the availability of femtosecond-pulse ultrafast lasers (with a pulse duration shorter than a phonon period), and techniques that can measure optical reflectivity with accuracy of one part in $10^{6}$. In most situations involving phonons, a classical description is adequate. However, at low enough temperatures, quantum fluctuations become dominant. For example, a recent study [3] shows that quantum fluctuations in the atomic positions can indeed influence observable quantities (e.g., the Raman line shape) even when temperatures are not very low. With these facts in mind, and prompted by the many exciting developments in classical phonon optics, coherent phonon experiments, and (on the other hand) squeezed states of light 洵, we would like to explore phonon analogs of quantum optics. In particular, we study the dynamical and quantum fluctuation properties of the atomic displacements, in analogy with the modulation of quantum noise in light. Specifically, we study single-mode and two-mode phonon coherent and squeezed states, and then focus on a polariton-based approach to achieve smaller quantum noise than the zero-point fluctuations of the atomic lattice. The concepts of coherent and squeezed states were both originally proposed in the context of quantum optics. A coherent state is a phasecoherent sum of number states. In this state, the quantum fluctuations in any pair of conjugate variables are at the lower limit of the Heisenberg uncertainty principle. In other words, a coherent state is as "quiet" as the vacuum state. Squeezed states [4] are interesting because they can have smaller quantum noise than the vacuum state in one of the conjugate variables, thus having a promising future in different applications ranging from gravitational wave detection to optical communications. In addition, squeezed states form an exciting group of states and can provide unique insight into quantum mechanical fluctuations. In recent years, squeezed states are also being explored in a variety of non-quantum-optics systems, including ion-motion and classical squeezing [5], molecular vibrations [6], polaritons [7,8], and phonons in crystals [9] 13]. Ref. [10 12] propose a second-order Raman scattering (SORS) process for phonon squeezing: if the two incident light beams are in coherent states, the phonons generated by the SORS are in a two-mode squeezed state. Here we first present an introduction to the subject of coherent and squeezed phonons, and later on consider both the continuous wave case studied in [10,12] and the impulsive case studied in 12, 14. Squeezed phonons could be detected by measuring the intensity of the reflected or transmitted probe light 10 12, 14. This method has been used to detect coherent phonon amplitudes, since reflectivity and transmission are closely related to the atomic displacements in a crystal. Measuring a transmitted probe light pulse, Ref. [14] observed 
squeezed phonons produced by an impulsive SORS. The intensity of the CW SORS signal for many materials might be too weak to be detected with current techniques, but might be accessible in the future.

\section{ANALOGIES AND DIFFERENCES BETWEEN PHONONS AND PHOTONS}

Coherent and squeezed states were initially introduced to describe photons. Here we are interested in applying these concepts to phonons. Although both photons and phonons are bosons, they do have significant differences, and the physics of squeezed states of light cannot be straightforwardly extended to phonons. Table I is a chart presenting a schematic comparison between phonons and photons. Below we briefly mention a few important similarities and differences that are relevant to our study.

Photons are elementary particles with no internal structure, thus are sometimes called simple bosons. On the other hand, phonons describe the collective displacements of very many atoms in a crystal, and are thus sometimes described as composite bosons [15]. Phonons are bosons because of the commutation relation between the coordinate and momentum operators. Kohn and Sherrington [15] pioneered the research on composite bosons like phonons, excitons, etc., and classified them into two categories, with type-I referring to those bosons composed of an even number of fermions (such as ${ }^{4} \mathrm{He}$ atoms), and type-II referring to those that are collective excitations - such as phonons, excitons, magnons, etc. In this sense it is also possible to consider photons to be type-II composite bosons 15, because they are the energy quanta of electromagnetic field modes. Their commutation relation originates from the simple harmonic oscillators that are used to quantize the electromagnetic field. Essentially, both photons and phonons are field quanta: photons are quanta of a continuous field, while phonons are quanta of a discrete field. Non-interacting phonons are used to describe harmonic crystal potentials. However, anharmonicity, which leads to phonon-phonon interactions, is always present. Some properties of solids, such as lattice heat conductivity and thermal expansion, solely depend on the anharmonic terms in the crystal potential. In other words, phonons in general interact with each other. For photons, the situation is somewhat different. In vacuum and at low intensity, photon interactions are so weak that the rule of linear superposition holds. However, in nonlinear media, photons are effectively interactive, with their interaction mediated by the atoms. As mentioned above, phonons exist in discrete media. Therefore, phonons have cut-off frequencies, which put an upper-limit to their energy spectra. For a diatomic lattice, this limit is of the order of $0.1 \mathrm{eV}$, which is in the infrared region. Photons, on the other hand, do not have such an upper bound for their energy. In addition, the discrete atomic lattice and the massive atoms lead to a finite zero-point fluctuation in the phonon field, while the continuous photon modes and the massless photons contribute to a divergent zero-point fluctuation in the photon field. The dispersion relations for photons and phonons are qualitatively different. Photons in free space have a linear dispersion relation. On the other hand, phonons have complicated nonlinear dispersion relations which generally have several acoustic and optical branches. The acoustic branches are linear around the center of the first Brillouin zone, i.e., the $\mathbf{k}=0$ point, which is at the continuum limit. When the quasi-wave-vector $\mathbf{k}$ is close to the first Brillouin zone boundary, $\omega$ saturates. The optical branches of the phonons have a different profile. Their dispersion relations are flat near $\mathbf{k}=0$, where $\omega=\omega_{0}$. Furthermore, as $\mathbf{k}$ increases, $\omega$ decreases; indeed, the optical phonon dispersion relation can be even more complicated depending on the lattice structure. Compared to photons, which generally have relatively simple dispersion relations, phonons have nonlinear dispersion relations that make it more difficult to satisfy both energy and momentum (in fact, quasi-momentum) conservation laws simultaneously. The order of magnitude of the crystal cohesion energy determines that phonons have very low energies. In addition, phonons can easily couple to many other excitations which are in a similar energy range, and be perturbed by thermal fluctuations even at low temperatures. All these couplings make phonon dynamics very dissipative. Due to its strong damping, coherent phonons have very short lifetimes ( 50 picosecond for optical phonons, while larger for acoustic phonons) [2]. On the other hand, there exist many materials in which photons can propagate with little dissipation, and furthermore very long photon coherent times can be produced by lasers. To summarize this brief comparison, we notice that the differences between phonons and photons can often hinder our effort to apply ideas originating in quantum optics to phonons. For example, there does not exist good phonon cavities at the moment, so that it is very difficult to select phonon modes. For phonons, we have to almost always deal with a continuum of phonon modes. In addition, short phonon lifetimes and the strongly dissipative environment of phonons also complicate the problems. These will be taken into consideration when we work on the theory. 


\section{PHONON OPERATORS AND THE PHONON VACUUM AND NUMBER STATES}

A phonon with quasimomentum $\mathbf{p}=\hbar \mathbf{q}$ and branch subscript $\lambda$ has energy $\epsilon_{\mathbf{q} \lambda}=\hbar \omega_{\mathbf{q} \lambda}$; the corresponding creation and annihilation operators satisfy the boson commutation relations

$$
\left[b_{\mathbf{q}^{\prime} \lambda^{\prime}}, b_{\mathbf{q} \lambda}^{\dagger}\right]=\delta_{\mathbf{q q}^{\prime}} \delta_{\lambda \lambda^{\prime}}, \quad\left[b_{\mathbf{q} \lambda}, b_{\mathbf{q}^{\prime} \lambda^{\prime}}\right]=0
$$

The atomic displacements $u_{i \alpha}$ of a crystal lattice are given by

$$
u_{i \alpha}=\frac{1}{\sqrt{N m}} \sum_{\mathbf{q} \lambda}^{N} U_{\mathbf{q} \alpha}^{\lambda} Q_{\mathbf{q}}^{\lambda} e^{i \mathbf{q} \cdot \mathbf{R}_{i}} .
$$

Here $\mathbf{R}_{i}$ refers to the equilibrium lattice positions, $\alpha$ to a particular direction, and $Q_{\mathbf{q}}^{\lambda}$ is the phonon normal-mode operator

$$
Q_{\mathbf{q}}^{\lambda}=\sqrt{\frac{\hbar}{2 \omega_{\mathbf{q} \lambda}}}\left(b_{\mathbf{q} \lambda}+b_{-\mathbf{q} \lambda}^{\dagger}\right) .
$$

For simplicity, hereafter we will drop the branch subscript $\lambda$, assume that $U_{\mathbf{q} \alpha}$ is real, and define a q-mode dimensionless lattice amplitude operator:

$$
u( \pm \mathbf{q})=b_{\mathbf{q}}+b_{-\mathbf{q}}^{\dagger}+b_{-\mathbf{q}}+b_{\mathbf{q}}^{\dagger} .
$$

This operator contains essential information on the lattice dynamics, including quantum fluctuations. It is the phonon analog of the electric field in the photon case. When no phonon is excited, the crystal lattice is in the phonon vacuum state $|0\rangle$. The expectation values of the atomic displacement and the lattice amplitude are zero, but the fluctuations will be finite:

$$
\begin{aligned}
\left\langle\left(\Delta u_{i \alpha}\right)^{2}\right\rangle_{\mathrm{vac}} & \equiv\left\langle\left(u_{i \alpha}\right)^{2}\right\rangle_{\mathrm{vac}}-\left\langle u_{i \alpha}\right\rangle_{\mathrm{vac}}^{2} \\
& =\sum_{\mathbf{q}}^{N} \frac{\hbar\left|U_{\mathbf{q} \alpha}\right|^{2}}{2 N m \omega_{\mathbf{q} \alpha}}, \\
\left\langle(\Delta u( \pm \mathbf{q}))^{2}\right\rangle_{\mathrm{vac}} & =2 .
\end{aligned}
$$

The eigenstates of the harmonic phonon Hamiltonian are number states which satisfy $b_{\mathbf{q}}\left|n_{\mathbf{q}}\right\rangle=\sqrt{n_{\mathbf{q}}}\left|n_{\mathbf{q}}-1\right\rangle$. The phonon number and the phase of atomic vibrations are conjugate variables. Thus, due to the uncertainty principle, the phase is arbitrary when the phonon number is certain, as it is the case with any number state $\left|n_{\mathbf{q}}\right\rangle$. Thus, in a number state, the expectation values of the atomic displacement $\left\langle n_{\mathbf{q}}\left|u_{i \alpha}\right| n_{\mathbf{q}}\right\rangle$ and $\mathbf{q}$-mode lattice amplitude $\left\langle n_{\mathbf{q}}|u( \pm \mathbf{q})| n_{\mathbf{q}}\right\rangle$ vanish due to the randomness in the phase of the atomic displacements. The fluctuations in a number state $\left|n_{\mathbf{q}}\right\rangle$ are

$$
\begin{aligned}
\left\langle\left(\Delta u_{i \alpha}\right)^{2}\right\rangle_{\text {num }} & =\frac{\hbar\left|U_{\mathbf{q} \alpha}\right|^{2} n_{\mathbf{q}}}{N m \omega_{\mathbf{q} \alpha}}+\sum_{\mathbf{q}^{\prime} \neq \mathbf{q}}^{N} \frac{\hbar\left|U_{\mathbf{q}^{\prime} \alpha}\right|^{2}}{2 N m \omega_{\mathbf{q}^{\prime} \alpha}}, \\
\left\langle(\Delta u( \pm \mathbf{q}))^{2}\right\rangle_{\mathrm{num}} & =2+2 n_{\mathbf{q}} .
\end{aligned}
$$

\section{PHONON COHERENT STATES}

A single-mode (q) phonon coherent state is an eigenstate of a phonon annihilation operator:

$$
b_{\mathbf{q}}\left|\beta_{\mathbf{q}}\right\rangle=\beta_{\mathbf{q}}\left|\beta_{\mathbf{q}}\right\rangle \text {. }
$$

It can also be generated by applying a phonon displacement operator $D_{\mathbf{q}}\left(\beta_{\mathbf{q}}\right)$ to the phonon vacuum state

$$
\begin{aligned}
\left|\beta_{\mathbf{q}}\right\rangle & =D_{\mathbf{q}}\left(\beta_{\mathbf{q}}\right)|0\rangle=\exp \left(\beta_{\mathbf{q}} b_{\mathbf{q}}^{\dagger}-\beta_{\mathbf{q}}^{*} b_{\mathbf{q}}\right)|0\rangle \\
& =\exp \left(-\frac{\left|\beta_{\mathbf{q}}\right|^{2}}{2}\right) \sum_{n_{\mathbf{q}}=0}^{\infty} \frac{\beta_{\mathbf{q}}^{n_{\mathbf{q}}}}{\sqrt{n_{\mathbf{q}} !}}\left|n_{\mathbf{q}}\right\rangle
\end{aligned}
$$


As is shown above, a phonon coherent state is a phase-coherent superposition of number states. Moreover, coherent states are a set of minimum-uncertainty states which are as noiseless as the vacuum state. Coherent states are also the set of quantum states that best describe the classical harmonic oscillators [16]. A single-mode phonon coherent state can be generated by the Hamiltonian

$$
H=\hbar \omega_{\mathbf{q}}\left(b_{\mathbf{q}}^{\dagger} b_{\mathbf{q}}+\frac{1}{2}\right)+\lambda_{\mathbf{q}}^{*}(t) b_{\mathbf{q}}+\lambda_{\mathbf{q}}(t) b_{\mathbf{q}}^{\dagger}
$$

and an appropriate initial state. Here $\lambda_{\mathbf{q}}(t)$ represents the interaction strength between phonons and the external source. More specifically, if the initial state is a vacuum state, $|\psi(0)\rangle=|0\rangle$, then the state vector becomes a single-mode coherent state thereafter

$$
|\psi(t)\rangle=\left|\Lambda_{\mathbf{q}}(t) e^{-i \omega_{\mathbf{q}} t}\right\rangle
$$

where

$$
\Lambda_{\mathbf{q}}(t)=-\frac{i}{\hbar} \int_{-\infty}^{t} \lambda_{\mathbf{q}}(\tau) e^{i \omega_{\mathbf{q}} \tau} d \tau
$$

is the coherent amplitude of mode $\mathbf{q}$. If the initial state is a single-mode coherent state $|\psi(0)\rangle=\left|\alpha_{\mathbf{q}}\right\rangle$, then the state vector at time $t$ takes the form

$$
|\psi(t)\rangle=\left|\left\{\Lambda_{\mathbf{q}}(t)+\alpha_{\mathbf{q}}\right\} e^{-i \omega_{\mathbf{q}} t}\right\rangle
$$

which is still a coherent state. In a single-mode $(\mathbf{q})$ coherent state $\left|\Lambda_{\mathbf{q}}(t) e^{-i \omega_{\mathbf{q}} t}\right\rangle,\left\langle u_{i \alpha}(t)\right\rangle_{\text {coh }}$ and $\langle u( \pm \mathbf{q})\rangle_{\text {coh }}$ are sinusoidal functions of time. The fluctuation in the atomic displacements is

$$
\left\langle\left(\Delta u_{i \alpha}\right)^{2}\right\rangle_{\mathrm{coh}}=\sum_{\mathbf{q}}^{N} \frac{\hbar\left|U_{\mathbf{q} \alpha}\right|^{2}}{2 N m \omega_{\mathbf{q} \alpha}}
$$

The unexcited modes are in the vacuum state and thus all contribute to the noise in the form of zero point fluctuations. Furthermore,

$$
\left\langle(\Delta u( \pm \mathbf{q}))^{2}\right\rangle_{\mathrm{coh}}=2
$$

¿From the expressions of the noise $\left\langle\left(\Delta u_{i \alpha}\right)^{2}\right\rangle_{\text {coh }}$ and $\left\langle(\Delta u[ \pm \mathbf{q})]^{2}\right\rangle_{\text {coh }}$, it is impossible to know which state (if any) has been excited, while this information is clearly present in the expression of the expectation value of the lattice amplitude $\langle u( \pm \mathbf{q})\rangle_{\text {coh }}$. These results can be straightforwardly generalized to multi-mode coherent states. Coherent phonons have been the subject of considerable interest in recent years. Typically, the dynamics of coherent phonons are described by using classical equations of motion. Here we present a quantum description and show that it is consistent with the classical one and, as an additional bonus, contains information on quantum fluctuations. Coherent phonons can be generated by a femtosecond short pulse laser [10]. A femtosecond pulse duration is much shorter than any phonon period and therefore acts as a delta-function driving force. It can produce coherent longitudinal optical (LO) phonons [2,10]. We can make a very simplified calculation by replacing the coupling strength $\lambda_{\mathbf{q}}(t)$ with $A \delta\left(t-t_{0}\right)$ in Eq. (13), so that

$$
H_{\mathrm{coh}}=\hbar \omega_{\mathbf{q}} b_{\mathbf{q}}^{\dagger} b_{\mathbf{q}}+A \delta\left(t-t_{0}\right) b_{\mathbf{q}}+A^{*} \delta\left(t-t_{0}\right) b_{\mathbf{q}}^{\dagger}
$$

Here $A=|A| e^{i \phi_{A}}$ is a time-independent complex amplitude containing the information of the photon-phonon interaction and the coherent amplitude of the relevant modes in the incident optical pulse. We assume that the crystal is in the phonon vacuum state before it is hit by the laser pulse at $t=t_{0}$. The time-evolution operator $U\left(t, t_{0}\right)$ for the phonon mode can be written as

$$
U\left(t, t_{0}\right)=\exp \left(-i \omega_{\mathbf{q}} t b_{\mathbf{q}}^{\dagger} b_{\mathbf{q}}\right) \exp \left(-\frac{i A}{2 \hbar} b_{\mathbf{q}} e^{-i \omega_{\mathbf{q}} t_{0}}-\frac{i A^{*}}{2 \hbar} b_{\mathbf{q}}^{\dagger} e^{i \omega_{\mathbf{q}} t_{0}}\right)
$$

In other words, for $t>t_{0}$, the crystal is in a coherent state $\left|\Lambda_{\mathbf{q}} e^{-i \omega_{\mathbf{q}} t}\right\rangle$. The coherent phonon amplitude $\Lambda_{\mathbf{q}}$ is

$$
\Lambda_{\mathbf{q}}=-\frac{i A^{*}}{2 \hbar} e^{i \omega_{\mathbf{q}} t_{0}}
$$


which is a constant complex number. Thus, a very short laser pulse conveniently provides a time-independent amplitude and a coherent phase to the active phonon mode(s). For $t>t_{0}$, the average of the atomic displacement operator in the state $\left|\Lambda_{\mathbf{q}} e^{-i \omega_{\mathbf{q}} t}\right\rangle \otimes\left|0_{-\mathbf{q}}\right\rangle$ becomes

$$
\begin{aligned}
\left\langle u_{i \alpha}\right\rangle & =\sqrt{\frac{\hbar}{2 N m \omega_{\mathbf{q}}}}\left(U_{\mathbf{q} \alpha} \Lambda_{\mathbf{q}} e^{-i \omega_{\mathbf{q}} t} e^{i \mathbf{q} \cdot \mathbf{R}_{i}}+U_{-\mathbf{q} \alpha} \Lambda_{\mathbf{q}}^{*} e^{i \omega_{\mathbf{q}} t} e^{-i \mathbf{q} \cdot \mathbf{R}_{i}}\right) \\
& =-\sqrt{\frac{2 \hbar}{N m \omega_{\mathbf{q}}}}\left|U_{\mathbf{q} \alpha} \Lambda_{\mathbf{q}}\right| \sin \left[\left(\omega_{q}\left(t-t_{0}\right)-\mathbf{q} \cdot \mathbf{R}_{i}-\phi_{A}-\phi_{U}\right] .\right.
\end{aligned}
$$

These longitudinal optical phonons can have a coherence time of about 50ps at $10 \mathrm{~K}$, and even longer at lower temperatures [2]. In the classical sense, "coherent" means a wave with a well-defined phase, or waves that can interfere with each other when superimposed. Here we have shown that a single-mode coherent state of phonons generated by a short laser pulse is indeed a plane wave with a well-defined phase. Thus, these phonons in a quantum coherent state are also coherent in a classical manner. Furthermore, they are in a minimum-uncertainty state.

\section{PHONON SQUEEZED STATES}

In order to reduce quantum noise to a level below the zero-point fluctuation level, we need to consider phonon squeezed states. Quadrature squeezed states are generalized coherent states [17. Here "quadrature" refers to the dimensionless coordinate and momentum. Compared to coherent states, squeezed ones can achieve smaller variances for one of the quadratures during certain time intervals and are therefore helpful for decreasing quantum noise. Figures 1 and 2 schematically illustrate several types of phonon states, including vacuum, number, coherent, and squeezed states. These figures are the phonon analogs of the illuminating schematic diagrams used for photons [17]. A single-mode quadrature phonon squeezed state is generated from a vacuum state as

$$
\left|\alpha_{\mathbf{q}}, \xi\right\rangle=D_{\mathbf{q}}\left(\alpha_{\mathbf{q}}\right) S_{\mathbf{q}}(\xi)|0\rangle ;
$$

a two-mode quadrature phonon squeezed state is generated as

$$
\left|\alpha_{\mathbf{q}_{1}}, \alpha_{\mathbf{q}_{2}}, \xi\right\rangle=D_{\mathbf{q}_{1}}\left(\alpha_{\mathbf{q}_{1}}\right) D_{\mathbf{q}_{2}}\left(\alpha_{\mathbf{q}_{2}}\right) S_{\mathbf{q}_{1}, \mathbf{q}_{2}}(\xi)|0\rangle .
$$

Here $D_{\mathbf{q}}\left(\alpha_{\mathbf{q}}\right)$ is the coherent state displacement operator with $\alpha_{\mathbf{q}}=\left|\alpha_{\mathbf{q}}\right| e^{i \phi}$,

$$
\begin{aligned}
S_{\mathbf{q}}(\xi) & =\exp \left(\frac{\xi^{*}}{2} b_{\mathbf{q}}^{2}-\frac{\xi}{2} b_{\mathbf{q}}^{\dagger 2}\right), \\
S_{\mathbf{q}_{1}, \mathbf{q}_{2}}(\xi) & =\exp \left(\xi^{*} b_{\mathbf{q}_{1}} b_{\mathbf{q}_{2}}-\xi b_{\mathbf{q}_{1}}^{\dagger} b_{\mathbf{q}_{2}}^{\dagger}\right),
\end{aligned}
$$

are the single- and two-mode squeezing operator, and $\xi=r e^{i \theta}$ is the complex squeezing factor with $r \geq 0$ and $0 \leq \theta<2 \pi$. The squeezing operator $S_{\mathbf{q}_{1}, \mathbf{q}_{2}}(\xi)$ can be produced by the following Hamiltonian:

$$
\begin{aligned}
H_{\mathbf{q}_{1}, \mathbf{q}_{2}}= & \hbar \omega_{\mathbf{q}_{1}} b_{\mathbf{q}_{1}}^{\dagger} b_{\mathbf{q}_{1}}+\hbar \omega_{\mathbf{q}_{2}} b_{\mathbf{q}_{2}}^{\dagger} b_{\mathbf{q}_{2}} \\
& +\zeta(t) b_{\mathbf{q}_{1}}^{\dagger} b_{\mathbf{q}_{2}}^{\dagger}+\zeta^{*}(t) b_{\mathbf{q}_{1}} b_{\mathbf{q}_{2}} .
\end{aligned}
$$

The time-evolution operator for such a Hamiltonian has the form

$$
U(t)=\exp \left(-\frac{i}{\hbar} H_{0} t\right) \exp \left[\xi^{*}(t) b_{\mathbf{q}_{1}} b_{\mathbf{q}_{2}}-\xi(t) b_{\mathbf{q}_{1}}^{\dagger} b_{\mathbf{q}_{2}}^{\dagger}\right]
$$

where

$$
\begin{aligned}
H_{0} & =\hbar \omega_{\mathbf{q}_{1}} b_{\mathbf{q}_{1}}^{\dagger} b_{\mathbf{q}_{1}}+\hbar \omega_{\mathbf{q}_{2}} b_{\mathbf{q}_{2}}^{\dagger} b_{\mathbf{q}_{2}}, \\
\xi(t) & =\frac{i}{\hbar} \int_{-\infty}^{t} \zeta(\tau) e^{i\left(\omega_{\mathbf{q}_{1}}+\omega_{\mathbf{q}_{2}}\right) \tau} d \tau .
\end{aligned}
$$

Here $\xi(t)$ is the squeezing factor and $\zeta(t)$ is the strength of the interaction between the phonon system and the external source; this interaction allows the generation and absorption of two phonons at a time. The two-mode phonon quadrature operators have the form 


$$
\begin{aligned}
X(\mathbf{q},-\mathbf{q}) & =2^{-3 / 2}\left(b_{\mathbf{q}}+b_{\mathbf{q}}^{\dagger}+b_{-\mathbf{q}}+b_{-\mathbf{q}}^{\dagger}\right) \\
& =2^{-3 / 2} u( \pm \mathbf{q}) \\
P(\mathbf{q},-\mathbf{q}) & =-i 2^{-3 / 2}\left(b_{\mathbf{q}}-b_{\mathbf{q}}^{\dagger}+b_{-\mathbf{q}}-b_{-\mathbf{q}}^{\dagger}\right) .
\end{aligned}
$$

We have considered two cases where squeezed states were involved in modes $\pm \mathbf{q}$. In the first case, the system is in a two-mode $( \pm \mathbf{q})$ squeezed state $\left|\alpha_{\mathbf{q}}, \alpha_{-\mathbf{q}}, \xi\right\rangle,\left(\xi=r e^{i \theta}\right)$, and its fluctuation is

$$
\left\langle[\Delta u( \pm \mathbf{q})]^{2}\right\rangle=2\left(e^{-2 r} \cos ^{2} \frac{\theta}{2}+e^{2 r} \sin ^{2} \frac{\theta}{2}\right) .
$$

In the second case, the system is in a single-mode squeezed state $\left|\alpha_{\mathbf{q}}, \xi\right\rangle\left(\alpha_{\mathbf{q}}=\left|\alpha_{\mathbf{q}}\right| e^{i \phi}\right)$ in the first mode and an arbitrary coherent state $\left|\beta_{-\mathbf{q}}\right\rangle$ in the second mode. The fluctuation is now

$$
\begin{aligned}
\left\langle[\Delta u( \pm \mathbf{q})]^{2}\right\rangle= & 1+e^{2 r} \sin ^{2}\left(\phi+\frac{\theta}{2}\right) \\
& +e^{-2 r} \cos ^{2}\left(\phi+\frac{\theta}{2}\right) .
\end{aligned}
$$

In both of these cases, $\left\langle[\Delta u( \pm \mathbf{q})]^{2}\right\rangle$ can be smaller than in coherent states (see Fig. 2).

\section{SECOND ORDER RAMAN SCATTERING (SORS)}

So far we have focused on concepts and the actual quantum mechanical states relevant to coherent and squeezed phonons. Now we will focus on one particular approach to generate squeezed phonon states via second order Raman scattering. The SORS process originates from the quadratic term in the polarizability change $\delta P_{\alpha \beta}$ of a crystal. The photon-phonon interaction $V$ that leads to the SORS process is [18] $V=-\frac{1}{4} \sum_{\alpha \beta} \sum_{\mathbf{q}}^{N} \sum_{j j^{\prime}} P_{\alpha \beta}^{\mathbf{q} j,-\mathbf{q} j^{\prime}} Q_{\mathbf{q} j} Q_{-\mathbf{q} j^{\prime}} E_{1 \alpha} E_{2 \beta}$. Here, $E_{1 \alpha}$ and $E_{2 \beta}$ are electric field amplitudes along $\alpha$ and $\beta$ directions with frequencies $\omega_{1}$ and $\omega_{2}$. The secondorder polarizability tensor $P_{\alpha \beta}^{\mathbf{q} j,-\mathbf{q} j^{\prime}}$ satisfies $P_{\alpha \beta}^{\mathbf{q} j,-\mathbf{q} j^{\prime}}=P_{\alpha \beta}^{-\mathbf{q} j^{\prime}, \mathbf{q} j}=P_{\alpha \beta}^{-\mathbf{q} j, \mathbf{q} j^{\prime}}$. Recall that the complex phonon normal mode operator $Q_{\mathbf{q} j}$ of the phonons is related to the phonon creation $b_{-\mathbf{q} j}^{\dagger}$ and annihilation $b_{\mathbf{q} j}$ operators by $Q_{\mathbf{q} j}=$ $b_{\mathbf{q} j}+b_{-\mathbf{q} j}^{\dagger}$. If the incident photon fields are not attenuated we can treat the optical fields as classical waves, and also consider the different pairs of $\pm \mathbf{q}$ modes as independent, and treat them separately. Thus, for one particular pair of $\pm \mathbf{q}$ modes, the complete Hamiltonian for the two phonon modes involved in the SORS process has the form [18]: $\mathcal{H}_{\mathbf{q}}=H_{\mathbf{q}}-\left\{4^{-1} \sum_{\alpha \beta} P_{\alpha \beta}^{\mathbf{q},-\mathbf{q}} E_{1 \alpha} E_{2 \beta}\right\} Q_{\mathbf{q}} Q_{-\mathbf{q}}$, where $H_{\mathbf{q}}=\hbar \omega_{\mathbf{q}}\left\{b_{\mathbf{q}}^{\dagger} b_{\mathbf{q}}+b_{-\mathbf{q}}^{\dagger} b_{-\mathbf{q}}\right\}$ is the free phonon Hamiltonian for the modes $\mathbf{q}$ and $-\mathbf{q}, \omega_{\mathbf{q}}=\left(\omega_{1}-\omega_{2}\right) / 2$, and the branch labels $j$ and $j^{\prime}$ have been dropped. Here we consider two different cases. The first is when the incident photons are in two monochromatic beams [10]12]; i.e., with electric fields $E_{j}=\mathcal{E}_{j} \cos \left(\omega_{j} t+\phi_{j}\right) ; j=1,2$. In the second case the incident photons are in an ultrashort pulse whose duration is much shorter than the phonon period 12,14 .

\section{SQUEEZED PHONONS VIA CONTINUOUS WAVE SORS}

Let us now first consider the continuous wave $(\mathrm{CW})$ case. Because the photons are monochromatic, we can take a rotating wave approximation [19] and keep only the on-resonance terms in the Hamiltonian. The off-resonance terms only contribute to virtual processes [20] at higher orders. This approximation is appropriate for times much longer than the phonon period. The simplified Hamiltonian has the form

$$
\begin{aligned}
\mathcal{H}_{\mathbf{q}}^{(c w)} & =H_{\mathbf{q}}-\lambda_{\mathbf{q}}\left\{b_{\mathbf{q}} b_{-\mathbf{q}} e^{2 i \omega_{\mathbf{q}} t+i \phi_{12}}+\text { c.c. }\right\} \\
\lambda_{\mathbf{q}} & =\frac{1}{16}\left|\sum_{\alpha \beta} P_{\alpha \beta}^{\mathbf{q},-\mathbf{q}} \mathcal{E}_{1 \alpha} \mathcal{E}_{2 \beta}\right|,
\end{aligned}
$$

where $\phi_{12}$ and $\lambda_{\mathbf{q}}$ refer to the overall phase and amplitude, respectively, of the product of the 2nd-order polarizability and the incident electric fields. Recall that $P_{\alpha \beta}^{\mathbf{q},-\mathbf{q}}$ is real, therefore the phase $\phi_{12}$ has no $\mathbf{q}$-dependence. It originates solely from the two photon modes. The Schrödinger equation for the $\pm \mathbf{q}$-mode phonons is $i \hbar \partial_{t}\left|\psi_{\mathbf{q}}(t)\right\rangle=$ 
$\mathcal{H}_{\mathbf{q}}^{(c w)}(t)\left|\psi_{\mathbf{q}}(t)\right\rangle$, and its time-evolution operator can be solved by a transformation into the interaction picture. The result can be expressed as 10,12.

$$
\left|\psi_{\mathbf{q}}(t)\right\rangle=e^{\left\{H_{\mathbf{q}} t / i \hbar\right\}} e^{\left\{\zeta_{\mathbf{q}}^{*} b_{\mathbf{q}} b_{-\mathbf{q}}-\zeta_{\mathbf{q}} b_{\mathbf{q}}^{\dagger} b_{-\mathbf{q}}^{\dagger}\right\}}\left|\psi_{\mathbf{q}}(0)\right\rangle,
$$

where $\zeta_{\mathbf{q}}=-i \lambda_{\mathbf{q}} t e^{-i \phi_{12} / \hbar}$. Notice that the second factor in the time-evolution operator is a two-mode quadrature squeezing operator [21]. In the $\mathrm{CW}$ case considered here, the amplitude of the squeezing factor $\zeta_{\mathbf{q}}$ grows linearly with time. However, this initial linear growth will be eventually curbed by subsequent phonon-phonon scattering and optical pump depletion. In other words, the expression for the squeezing factor $\zeta_{\mathbf{q}}$ is valid for times much larger than one phonon period, but much smaller than phonon lifetimes (because this treatment considers non-decaying phonons). By solving the phonon Langevin equation, we have shown that the squeezing factor in the CW SORS will eventually saturate at a constant value determined by the strength of SORS and the phonon decay constant [22]. In addition, the phase of the squeezing factor is determined by the phase difference of the two incoming light waves. If the $\pm \mathbf{q}$ phonon modes are initially in a vacuum state or in a coherent state, the SORS will drive them into a two-mode quadrature squeezed state [10,12]. The time evolution operator of all the phonon mode pairs (instead of just one pair of $\pm \mathbf{q}$ modes) that are involved in this SORS process has the form $U(t)=\prod_{\mathbf{q}} U_{\mathbf{q}}(t)$. Therefore, as long as the photon depletion is negligible, all the phonon modes that are involved in a SORS process are driven into two-mode quadrature squeezed states. In other words, squeezing can be achieved in a continuum of phonon modes by a CW stimulated SORS process.

\section{SQUEEZED PHONONS VIA IMPULSIVE SORS}

Recently, an impulsive SORS process has been used to experimentally generate phonon squeezing [14. Here we treat the problem expressing the time evolution operator of the system in terms of a product of the two-mode quadrature squeezing operator and the free rotation operators [23]. Since the incident photons are now in an ultrashort pulse, the complete Hamiltonian can be solved in the limit when the optical field can be represented by a $\delta$-function. Such an approximation is usually considered when the optical pulse duration is much shorter than the optical phonon period, which is experimentally feasible with femtosecond laser pulses. The Hamiltonian for the SORS can now be written as $\mathcal{H}^{\prime}=\sum_{\mathbf{q}}\left\{H_{\mathbf{q}}-\lambda_{\mathbf{q}}^{\prime} \delta(t) Q_{\mathbf{q}} Q_{-\mathbf{q}}\right\}$, where $\lambda_{\mathbf{q}}^{\prime}$ carries the information on the amplitudes of the incoming optical fields and the electronic polarizability. Notice that the light-phonon coupling strength $\lambda_{\mathbf{q}}$ in the CW case has units of energy, while $\lambda_{\mathbf{q}}^{\prime}$ here has units of $\hbar$. To further simplify the problem, we assume that only $\pm \mathbf{q}$ modes are involved in the process. Such a simplification is possible when the photon depletion and the phonon anharmonic interaction are negligible, so that different pairs of phonon modes are independent from each other. The Hamiltonian is now

$$
\mathcal{H}_{\mathbf{q}}^{\prime}=H_{\mathbf{q}}-\lambda_{\mathbf{q}}^{\prime} \delta(t) Q_{\mathbf{q}} Q_{-\mathbf{q}}
$$

and the Schrödinger equation for these two phonon modes is $i \hbar \partial_{t}\left|\psi_{\mathbf{q}}(t)\right\rangle=\mathcal{H}_{\mathbf{q}}^{\prime}\left|\psi_{\mathbf{q}}(t)\right\rangle$. This equation can be solved by separating the free oscillator terms and the two-phonon creation and annihilation terms. The resulting time-dependent wavefunction is

$$
\begin{aligned}
\left|\psi_{\mathbf{q}}(t)\right\rangle= & \exp \left\{\frac{t H_{\mathbf{q}}}{i \hbar}\right\} \exp \left\{\frac{i \lambda_{\mathbf{q}}^{\prime} H_{\mathbf{q}}}{\hbar^{2} \omega_{\mathbf{q}}}\right\} \\
& \times \exp \left\{\zeta_{\mathbf{q}}{ }^{*} b_{\mathbf{q}} b_{-\mathbf{q}}-\zeta_{\mathbf{q}}^{\prime} b_{\mathbf{q}}^{\dagger} b_{-\mathbf{q}}^{\dagger}\right\}\left|\psi_{\mathbf{q}}\left(0^{-}\right)\right\rangle .
\end{aligned}
$$

Here $\zeta_{\mathbf{q}}^{\prime}=-i \lambda_{\mathbf{q}}^{\prime} e^{-i \lambda_{\mathbf{q}}^{\prime} / \hbar} / \hbar$. Hence the effect of the optical pulse is clear: it first applies a two-mode quadrature squeezing operator on the initial state, then rotates the state by changing its phase [23]. The state will then freely evolve after $t=0^{+}$. This result is consistent with Ref. [14] where the time-evolution operator is expressed in terms of real phonon normal mode operators [18], instead of the complex ones used in this paper. Notice that, in contrast to the CW SORS, the phase of the squeezing factor $\zeta^{\prime}$ for the impulsive case is fixed by the intensity of the light pulse.

\section{MACROSCOPIC IMPLICATIONS AND TIME-DEPENDENCE OF THE DIELECTRIC CONSTANT}

Now that we have obtained the phonon states for both the CW and pulsed SORS cases, let us consider the macroscopic implications of these states. More generally, let us first discuss the implications of the phonon squeezed 
states disregarding how they are generated. An experimentally observable quantity $O$ which is related to the atomic displacements in the crystal can generally be expressed in terms of $Q_{\mathbf{q}}: O=O(0)+\sum_{\mathbf{q}}\left(\partial O / \partial Q_{\mathbf{q}}\right) Q_{\mathbf{q}}+\ldots=O_{0}+$ $O_{1}+O_{2}+\ldots$ where the first term $O_{0}=O(0)$ is the operator $O$ when all $Q_{\mathbf{q}}$ 's vanish. An example of an experimentally observable quantity $O$ is the change in the crystal dielectric constant $\delta \epsilon$ due to the atomic displacement produced by the incident electric fields. To first order in $\left.Q_{\mathbf{q}}, \delta \epsilon=\delta \epsilon_{1}=\sum_{q_{x}>0}\left|\frac{\partial(\delta \epsilon)}{\partial Q_{\mathbf{q}}}\right| \sqrt{\frac{\hbar}{2 \omega_{\mathbf{q}}}}\left(b_{\mathbf{q}}+b_{-\mathbf{q}}^{\dagger}\right) e^{i \Psi_{\mathbf{q}}}+\left(b_{-\mathbf{q}}+b_{\mathbf{q}}^{\dagger}\right) e^{-i \Psi_{\mathbf{q}}}\right]$. Here $\Psi_{\mathbf{q}}$ is the phase of $\partial O / \partial Q_{\mathbf{q}}=\partial(\delta \epsilon) / \partial Q_{\mathbf{q}}$. Indeed, a widely used method to track the phases of coherent phonons in the time-domain [2] is based on the observation of the reflectivity (or transmission) modulation $\delta R(\delta T)$ of the sample, which is linearly related to $\delta \epsilon$ - the change in the dielectric constant due to lattice vibrations. The above equation for $\delta \epsilon$ indicates that we can define a generalized [10] lattice amplitude operator [8,11]: $u_{g}( \pm \mathbf{q})=$ $\left(b_{\mathbf{q}}+b_{-\mathbf{q}}^{\dagger}\right) e^{i \Psi_{\mathbf{q}}}+\left(b_{-\mathbf{q}}+b_{\mathbf{q}}^{\dagger}\right) e^{-i \Psi_{\mathbf{q}}}$. This generalized lattice amplitude $u_{g}( \pm \mathbf{q})=2 \operatorname{Re}\left\{Q_{\mathbf{q}} e^{i \Psi_{\mathbf{q}}}\right\}$ is the underlying microscopic quantity related to an observed reflectivity or transmission modulation when the linear term in $Q_{\mathbf{q}}, \delta \epsilon_{1}$, exists. Even if $\left\langle\delta \epsilon_{1}\right\rangle$ vanishes, $\left\langle\Delta\left(\delta \epsilon_{1}\right)^{2}\right\rangle$ does not. Since different pairs of $\pm \mathbf{q}$ phonon modes are uncorrelated to one another, the fluctuation of $\delta \epsilon_{1}$ can be expressed as $\left\langle\left(\Delta \delta \epsilon_{1}\right)^{2}\right\rangle=\sum_{q_{x}>0}\left(\hbar / 2 \omega_{\mathbf{q}}\right)\left|\partial(\delta \epsilon) / \partial Q_{\mathbf{q}}\right|^{2}\left\langle\Delta u_{g}^{2}( \pm \mathbf{q})\right\rangle$. Here the state is $|\psi(t)\rangle=U(t)|\psi(0)\rangle=\prod_{\mathbf{q}} U_{\mathbf{q}}(t)\left|\psi_{\mathbf{q}}(0)\right\rangle$ in either the $\mathrm{CW}$ or the impulsive case. We can again focus on a single pair of $\pm \mathbf{q}$ modes. In the CW case, using Eq. (36), the fluctuation is

$$
\begin{aligned}
\left\langle\Delta u_{g}^{2}( \pm \mathbf{q})\right\rangle^{(c w)}= & 2\left\{e^{-2 r_{\mathbf{q}}} \cos ^{2}\left(\Omega_{\mathbf{q}}(t)+\phi_{12} / 2\right)\right. \\
& \left.+e^{2 r_{\mathbf{q}}} \sin ^{2}\left(\Omega_{\mathbf{q}}(t)+\phi_{12} / 2\right)\right\},
\end{aligned}
$$

where $r_{\mathbf{q}}=\left|\zeta_{\mathbf{q}}\right|=\lambda_{\mathbf{q}} t / \hbar, \Omega_{\mathbf{q}}(t)=\omega_{\mathbf{q}} t+\pi / 4$, and hereafter $\langle\ldots\rangle$ denotes an expectation value on squeezed states, unless stated otherwise. Therefore, at certain times, the fluctuation $\left\langle\Delta u_{g}^{2}( \pm \mathbf{q})\right\rangle^{(c w)}$ can be smaller than 2, which is the vacuum fluctuation level. Furthermore, all the pairs of phonon modes that are driven by the stimulated SORS process share the same frequency: $\omega_{\mathbf{q}}=\left(\omega_{1}-\omega_{2}\right) / 2$. Therefore, all the fluctuations $\left\langle\Delta u_{g}^{2}( \pm \mathbf{q})\right\rangle^{(c w)}$ evolve with the same $\omega_{\mathbf{q}}$. Notice that there is no dependence on $\Psi_{\mathbf{q}}$ in the final expression of $\left\langle\Delta u_{g}^{2}( \pm \mathbf{q})\right\rangle^{(c w)}$, and the squeezing factor phase $\phi_{12} / 2$ has no $\mathbf{q}$-dependence, all the pairs of modes involved through the SORS share the same phase in their fluctuations. Therefore there can be squeezing in the overall fluctuation $\left\langle\left(\Delta \delta \epsilon_{1}\right)^{2}\right\rangle^{(c w)}$. Furthermore, the phase of this overall fluctuation can be adjusted by tuning the phase difference of the two incoming light beams. In the impulsive case [14,2], if the $\pm \mathbf{q}$-mode phonons are driven into a squeezed vacuum state, the fluctuation in $u_{g}( \pm \mathbf{q})$ is

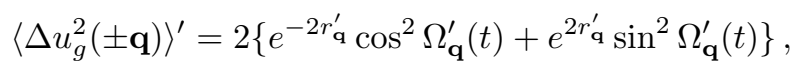

where $r_{\mathbf{q}}^{\prime}=\left|\zeta_{\mathbf{q}}^{\prime}\right|=\lambda_{\mathbf{q}}^{\prime} / \hbar$, and $\Omega_{\mathbf{q}}^{\prime}(t)=\Omega_{\mathbf{q}}(t)-r_{\mathbf{q}}^{\prime}$. Again, the squeezing will reveal itself through oscillations in $\left\langle\left[\Delta\left(\delta \epsilon_{1}\right)\right]^{2}(\mathbf{q})\right\rangle^{\prime}$ which is proportional to $\left\langle\Delta u_{g}^{2}( \pm \mathbf{q})\right\rangle^{\prime}$. Note that these oscillations are essentially the same as the ones obtained in the CW case. However, now the squeezing factor is time-independent. Also, the $t=0$ phase $\pi / 4-r_{\mathbf{q}}^{\prime}$ in Eq. (40) is q-dependent. Eq.(40) can be rewritten as $\left\langle\Delta u_{g}^{2}( \pm \mathbf{q})\right\rangle^{\prime}=2\left\{\cosh 2 r_{\mathbf{q}}^{\prime}+\sinh 2 r_{\mathbf{q}}^{\prime} \sin \left(2 \omega_{\mathbf{q}} t-r_{\mathbf{q}}^{\prime}\right)\right\}$. For small $r_{\mathbf{q}}^{\prime}$, this becomes $\left\langle\Delta u_{g}^{2}( \pm \mathbf{q})\right\rangle^{\prime}=2\left\{1+2 r_{\mathbf{q}}^{\prime 2}+2 r_{\mathbf{q}}^{\prime} \sin \left(2 \omega_{\mathbf{q}} t-r_{\mathbf{q}}^{\prime}\right)\right\}$. This expression has essentially the same form as the one obtained in [14]: $\left\langle Q_{\mathbf{q}}^{2}(t)\right\rangle=\left\langle Q_{\mathbf{q}}^{2}(0)\right\rangle\left\{1+2 \xi_{\mathbf{q}}^{2}+2 \xi_{\mathbf{q}} \sin \left(2 \omega_{\mathbf{q}} t+\varphi_{\mathbf{q}}\right)\right\}$. The small phase term $\varphi_{\mathbf{q}}$ is neglected in [14] when computing transmission changes. The difference in phases, $r_{\mathbf{q}}^{\prime}$ versus $\varphi_{\mathbf{q}}$, is negligible in the limit of very small squeezing factor, and originates from the different interaction Hamiltonians used here and in [14]. The interaction term in 14] is proportional to $u_{g}^{2}( \pm \mathbf{q})$ with $\Psi_{\mathbf{q}}=0$ (notice that their $Q_{\mathbf{q}}$ is real and based on standing wave quantization [18]). Therefore, the interaction Hamiltonian in [14] is (in our notation) $V \propto u_{g}^{2}( \pm \mathbf{q}) \propto 2 Q_{\mathbf{q}} Q_{-\mathbf{q}}+Q_{\mathbf{q}}^{2}+Q_{-\mathbf{q}}^{2}$. However, the last two terms in this expression do not satisfy momentum conservation, we thus did not include them and kept only $Q_{\mathbf{q}} Q_{-\mathbf{q}}$ in our interaction term (this form is also used by Ref. [181). When the linear perturbation $\delta \epsilon_{1}$ due to phonons vanishes, such as in 14, the second order correction $O_{2}\left(=\delta \epsilon_{2}\right)$ must be considered. When the phonon states are modulated by a SORS, so that the $\pm \mathbf{q}$ modes are the only ones which are correlated, $\delta \epsilon_{2}=\sum_{\mathbf{q}} \frac{\partial^{2}(\delta \epsilon)}{\partial Q_{\mathbf{q}} \partial Q_{-\mathbf{q}}} Q_{\mathbf{q}} Q_{-\mathbf{q}}$. Let us first focus on one pair of $\pm \mathbf{q}$ modes in the CW case. In a vacuum state, $\left\langle 0\left|Q_{\mathbf{q}} Q_{-\mathbf{q}}\right| 0\right\rangle=1$; while in a squeezed vacuum state $|0\rangle_{\mathrm{sq}}$, sq $\left\langle 0\left|Q_{\mathbf{q}} Q_{-\mathbf{q}}\right| 0\right\rangle_{\mathrm{sq}}=\left\langle\Delta u_{g}^{2}( \pm \mathbf{q})\right\rangle^{(c w)} / 2$, with the right hand side given in Eq. (39). Therefore, the expectation value of $Q_{\mathbf{q}} Q_{-\mathbf{q}}$ in a squeezed vacuum state is periodically smaller than its vacuum state value. Let us now include all the phonon modes that contribute to $\delta \epsilon_{2}$. In a vacuum state, $\left\langle 0\left|\delta \epsilon_{2}\right| 0\right\rangle=\sum_{\mathbf{q}} \partial^{2} \delta \epsilon /\left(\partial Q_{\mathbf{q}} \partial Q_{-\mathbf{q}}\right)$. On the other hand, in a squeezed vacuum state,

$$
\left\langle\delta \epsilon_{2}\right\rangle=\frac{1}{2} \sum_{\mathbf{q}} \frac{\partial^{2}(\delta \epsilon)}{\partial Q_{\mathbf{q}} \partial Q_{-\mathbf{q}}}\left\langle\Delta u_{g}^{2}( \pm \mathbf{q})\right\rangle^{(c w)} .
$$

Since the phase $\phi_{12} / 2$ has no $\mathbf{q}-$ dependence, contributions from the phonon modes sharing the same frequency add up constructively. It is thus possible that $\left\langle\delta \epsilon_{2}\right\rangle$ is periodically smaller than its vacuum state value. Similarly, in 
the impulsive case, $\left\langle\delta \epsilon_{2}\right\rangle^{\prime}=2^{-1} \sum_{\mathbf{q}} \frac{\partial^{2}(\delta \epsilon)}{\partial Q_{\mathbf{q}} \partial Q_{-\mathbf{q}}}\left\langle\Delta u_{g}^{2}( \pm \mathbf{q})\right\rangle^{\prime}$; However, the phase factor in $\left\langle\delta \epsilon_{2}\right\rangle^{\prime}$ has a $\mathbf{q}-$ dependence through $r_{\mathbf{q}}^{\prime}$, so that all the phonon modes with the same $\omega_{\mathbf{q}}$ do not contribute to $\left\langle\delta \epsilon_{2}\right\rangle^{\prime}$ synchronously. In the CW SORS and in the very-small- $r_{\mathbf{q}}^{\prime}$ limit impulsive SORS the phase of the expectation value $\left\langle Q_{\mathbf{q}} Q_{-\mathbf{q}}\right\rangle$ does not depend on $\mathbf{q}$; this is crucial to the experimental observation of modulations in the dielectric constant, because this q-insensitivity leads to constructive summations of all the $\mathbf{q}$ pairs involved. Also, at a van Hove singularity a large number of modes contribute to $\delta \epsilon_{2}$ with the same frequency and phase, thus their effect is larger and easier to observe [14].

\section{SQUEEZED PHONONS VIA A FINITE-WIDTH SORS}

Real light pulses are not $\delta$-functions. Therefore, we have also considered a SORS pumped by a light pulse with a finite width (smaller than the phonon period $T$ ) instead of a $\delta$-function. For a fixed peak height $I$, we find [22] that the optimal pulse width $T_{\mathrm{p}}^{\mathrm{opt}}$ that maximizes the squeezing effect satisfies $T_{\mathrm{p}}^{\mathrm{opt}} \approx T / 4.4$. This calculation indicates that the experiments [14] used a pulse width which is nearby the optimal value $\left(T / 4.4 \approx 300 / 4.4 \mathrm{fs} \approx 68 \mathrm{fs} \approx T_{\mathrm{p}}\right)$. The calculation [22] can be summarized as follows. First, in the impulsive Hamiltonian we replace the $\delta$-function by a Gaussian with its width $T_{\mathrm{p}}$ as a variational parameter. Since now the Hamiltonian is time-dependent in the interaction picture, we cannot directly integrate the Schrödinger equation. Instead, we use the Magnus method to obtain the time evolution operator and keep only the dominant first term. This approximation is valid when the pulse duration is shorter than the phonon period. We then calculate the width $T_{\mathrm{p}}^{\mathrm{opt}}$ of the Gaussian that maximizes the squeezing factor. For a constant peak intensity, a pulse that is too narrow does not contain enough photons; while it can be proven that a pulse which is too long (i.e., with a width comparable to $T$ ), attenuates the squeezing effect.

\section{PHONON SQUEEZING MECHANISM}

What is the mechanism of phonon squeezing in the SORS processes? For the CW case, the Hamiltonian is the same as an optical two-mode parametric process 19], with the low frequency interference of the combined photon modes as the pump, the two phonon modes as the signal and idler. The frequencies of these modes satisfy $\omega_{\mathbf{q}}+\omega_{-\mathbf{q}}=\omega_{1}-\omega_{2}$. The impulsive case is slightly different. Although the Hamiltonian is similar to a parametric process, the energy transfer from the photons to the two phonon modes is instantaneous. The resulting phonon state is a two-mode quadrature squeezed vacuum state. Indeed, a regular parametric process pumps energy into the signal and idler modes gradually, while the impulsive SORS does it suddenly. The correlation between the two phonon modes, and thus the squeezing effect, is also introduced instantaneously. Notice that this mechanism is reminiscent of the frequency-jump mechanism proposed in [6]. In the impulsive SORS, the frequency of the phonon modes has an "infinite" $\delta$-peak change at $t=0$, while the

frequency-jump mechanism has finite frequency changes, and squeezing there can be intensified by repeated frequency jumps at appropriate times. However, as it has been pointed out in [6], a finite frequency jump up immediately followed by an equal jump down results in no squeezing at all.

\section{EXPERIMENTAL SEARCH FOR SQUEEZED PHONONS}

One group [14] has claimed to detect squeezed phonons. Regrettably, no other group has succeeded in reproducing their results. Recent calculations indicate that these initial experiments [14 do not prove that phonon squeezing has been achieved experimentally. Here we discuss several important points to be considered for the eventual future experimental observation of a phonon squeezed state. First of all, squeezing must refer to a phonon mode with a variance that falls below the standard quantum limit. This crucial comparison has not been produced yet. Thus, a reliable determination of the vacuum noise level is necessary in order to establish if a squeezing of the vacuum noise has indeed occurred. Experiments in quantum optics have an independent way to reliably obtain the noise level of the vacuum: by using an independent light beam as a local oscillator. Currently, there is no such phase-sensitive detection scheme for phonons. However, as we point out later, a pump-probe experiment may be able to establish such a criteria, but discretion has to be applied. Second, for phonons, with relatively low energy compared to photons, thermal noise should always be considered in an experiment. This is especially important when the noise modulation factor is small, such as the case in the reported experiment [14], where the noise modulation factor is only $0.0001 \%$. To achieve total noise modulation, as can be done through CW and impulsive SORS, is not equivalent to the squeezing of the vacuum 
noise. Only when the noise modulation factor is big enough to overcome the thermal noise, then the quantum noise is suppressed. Third, in that experiment, the squeezing factor is obtained from the modulation of the light transmission through a crystal. However, this change in light transmission describes the modulation of the total fluctuations (both quantum and thermal) of the atomic displacements. Thus, by itself this modulation of the noise is not a proof of quantum noise suppression, let alone of squeezing below the vacuum noise level. Fourth, the efficiency of the signal detection is a crucial issue in photon squeezing experiments, but not addressed in its phonon "analog" experiment. In a homodyne or heterodyne detector for photons, if the efficiency of the photon counters is less than one, additional noise is introduced into the signal. Similarly, in a pump-probe phonon detection scheme, the probe light pulse and photodetector should introduce additional noise into the final signal. Therefore, a careful analysis of these additional noise sources should be performed when employing a pump-probe scheme to detect phonon squeezing. For the above reasons, it is premature and unwarranted to claim that squeezed phonons have been observed experimentally. The evidence presented so far is incomplete and inconclusive. The answer to the question: "Can phonons be squeezed like photons?" is yes on theoretical grounds, but the experimental proof still lies in the future. The rest of this section presents quantitative derivations in support of the statements made above. Even with the reservations presented above regarding the pump-probe scheme, we would like to point out that it does provide a possible means for the detection of phonon squeezing. Here we discuss the criteria for achieving squeezing of quantum noise through an impulsive SORS process in a pump-probe experiment. We then give a numerical estimate on whether squeezing of quantum noise has been achived in [14]. Experimentally, the observed quantity is the change in the transmission $T$ due to the impulsive SORS process. Up to second order in $Q_{\mathbf{q}}, T$ can be expressed as

$$
T=T_{0}+\sum_{\mathbf{q}} \frac{\partial T}{\partial Q_{\mathbf{q}}}\left\langle Q_{\mathbf{q}}\right\rangle+\sum_{\mathbf{q}} \frac{\partial^{2} T}{\partial Q_{\mathbf{q}} \partial Q_{\mathbf{q}^{\prime}}}\left\langle Q_{\mathbf{q}} Q_{\mathbf{q}^{\prime}}\right\rangle
$$

where the average is over the phonon states of the crystal. In a squeezed vacuum (or thermal) state considered here here, $\left\langle Q_{\mathbf{q}}\right\rangle=0$ and $\left\langle Q_{\mathbf{q}} Q_{-\mathbf{q}}\right\rangle=2\left\langle\Delta u^{2}( \pm \mathbf{q}, t)\right\rangle$. Therefore, $T$ does contain informations on the atomic lattice fluctuations. Through impulsive SORS, we can achieve modulation of the total noise of the system, and the effect can be seen through a modulation of the transmission $T$, or more specifically, $\Delta T / T$. Starting from a thermal state, we have:

$$
\frac{\Delta T}{T} \approx \frac{1}{T} \sum_{\mathbf{q}} \frac{\partial^{2} T}{\partial Q_{\mathbf{q}} \partial Q_{-\mathbf{q}}}\left[1+2 N\left(\omega_{\mathbf{q}}\right)\right] r_{\mathbf{q}} \sin \left(2 \omega_{\mathbf{q}} t\right)
$$

Notice that this modulation of the total noise is not equivalent to a squeezing of quantum noise, because $\Delta T=0$ is an indication of total noise limit (That is, quantum noise plus thermal noise), not quantum noise limit. Even though the experiment is done at low temperatures, so that thermal noise is miniscule, we still have to compare the thermal noise to the squeezing factor (or modulation factor through impulsive SORS) because the later is very small, too. One criteria for quantum noise suppression would be

$$
\begin{aligned}
\frac{\Delta T^{\prime}}{T} & =\frac{T-T_{\mathrm{vac}}}{T_{\mathrm{vac}}} \\
& \approx \frac{1}{T_{\mathrm{vac}}} \sum_{\mathbf{q}} \frac{\partial^{2} T}{\partial Q_{\mathbf{q}} \partial Q_{-\mathbf{q}}}\left[2 N\left(\omega_{\mathbf{q}}\right)+r_{\mathbf{q}} \sin \left(2 \omega_{\mathbf{q}} t\right)\right] .
\end{aligned}
$$

A negative $\Delta T^{\prime} / T$ here indicates that, at least in some spectral regions, the atomic displacement noise is squeezed below the ground state limit. We are now comparing the thermal population $2 N\left(\omega_{\mathbf{q}}\right)$ and the squeezing factor $r_{\mathbf{q}}$. In summary, there is always modulation of total noise by the impulsive SORS, but only when $r_{\mathbf{q}}>2 N\left(\omega_{\mathbf{q}}\right)$ do we achieve noise smaller than the ground state limit. For example, at an experimental temperature of $10 \mathrm{~K}$, the corresponding thermal energy $k_{B} T$ is about $0.88 \mathrm{meV}$. The acoustic phonons involved in the experiment reported in [14] have a frequency of about $2.7 \mathrm{THz}$, which corresponds to an energy quantum of $\hbar \omega \approx 10.6 \mathrm{meV}$. Therefore, the thermal noise factor here is about $N=1 /\left\{\exp \left(\hbar \omega / k_{B} T\right)-1\right\} \approx e^{-12} \approx 0.6 * 10^{-5}$, which is almost identical to the squeezing factor given in 14]. Therefore, it is quite clear that this particular experiment has not achieved squeezing of quantum noise below the vacuum noise limit. However, if the experimental temperature is further lowered, the thermal factor will become smaller. The squeezing action initiated by the second order Raman scattering should then be strong enough to realize suppression of noise below the ground state level. 


\section{CONCLUSIONS}

We have presented an overview of the definitions relevant to quantum phonon optics, including quantum coherent and squeezed phonons. Afterwards, we have studied theoretically the generation of phonon squeezing using a stimulated SORS process. In particular, we calculated the time evolution operators of the phonons in two different cases: when the incident photons are in monochromatic continuous waves, and when they are in an ultrashort pulse. The amplitude of the squeezing factor initially increases with time and then saturates in the CW SORS case, while it remains constant in the pulsed SORS case. In addition, the $t=0$ phase of the squeezing factor in the CW SORS, $\phi_{12}$, can be continuously adjusted by tuning the relative phase of the two incoming monochromatic photon beams, while for the pulsed SORS the phase $\left(\propto \lambda_{\mathbf{q}}^{\prime}\right)$ of the squeezing factor is determined by the amplitude of the incoming light pulse. For both cases we calculated the quantum fluctuations of a generalized lattice amplitude operator and the second order contribution to the change in dielectric constant, which is measurable. For the finite-width impulsive case, we computed the optimal pulse width, in terms of the phonon period, that maximizes the squeezing effect.

\section{ACKNOWLEDGEMENTS}

We acknowledge useful conversations with S. Hebboul, S. Tamura and H. Wang. One of us (XH) acknowledges support from the US Army Research Office.

[1] V. Narayanamurti et al., Phys. Rev. Lett. 43, 2012 (1979); C.F. Quate, Sci. Am. 241, 62 (1979); J.P. Wolfe, Phys. Today 33(12), 44 (1980); V. Narayanamurti, Science 213, 717 (1981); S. Tamura, Phys. Rev. B 30, 849 (1984); G.A. Northrop, S.E. Hebboul, and J.P. Wolfe, Phys. Rev. Lett. 55, 95 (1985); S. Tamura and F. Nori, Phys. Rev. B 38, 5610 (1988); S.E. Hebboul and J.P. Wolfe, Z. Phys. B 73, 437 (1989); S. Tamura, in Phonon 89, edited by S. Hunkulinger, W. Ludwig, and G. Weiss (World Scientific, Singapore, 1989), p. 703; S. Tamura and F. Nori, Phys. Rev. B 41, 7941 (1990); N. Nishiguchi, S. Tamura, and F. Nori, Phys. Rev. B 48, 2515 (1993); ibid., 14426 (1993).

[2] For excellent reviews on coherent phonons, see, e.g., S. Ruhman, A.G. Joly and K. Nelson, IEEE J. Quantum Electron. 24, 460 (1988); W.A. Kütt, W. Albrecht and H. Kurz, ibid. 28, 2434 (1992).

[3] F. Nori, R. Merlin, S. Haas, A.W. Sandvik and E. Dagotto, Phys. Rev. Lett. 75, 553 (1995).

[4] See, e.g., the special issue on squeezed states, Appl. Phys. B 55, No.3 (1992).

[5] J. Sidles and D. Rugar, Phys. Rev. Lett. 70, 3506 (1993); V. Natarajan et al. 74, 2855 (1995); J.I. Cirac et al. 70, 556 (1993).

[6] J. Janszky and P. Adam, Phys. Rev. A 46, 6091 (1992); J. Janszky et al., Spectrochim. Acta A 48, 31 (1992); J. Janszky and An. V. Vinogradov, Phys. Rev. Lett. 64, 2771 (1990).

[7] M. Artoni and J.L. Birman, Optics Comm. 89, 324 (1992); 104, 319 (1994).

[8] X. Hu and F. Nori, Phys. Rev. B 53, 2419 (1996).

[9] X. Hu and F. Nori, Bull. Am. Phys. Soc. 39, 466 (1994); 41, 657 (1996).

[10] X. Hu and F. Nori, UM preprint 8-15-95 (1995); X. Hu, PhD Thesis, Univ. of Michigan, 1996 (Univ. Microfilm Intern.).

[11] X. Hu and F. Nori, Phys. Rev. Lett. 76, 2294 (1996).

[12] X. Hu and F. Nori, Phys. Rev. Lett. 79, 4605 (1997).

[13] P.F. Schewe and B. Stein, AIP Physics News, 261 (3-6-1996).

[14] G. Garett et al., Science 275, 1638 (1997).

[15] W. Kohn and D. Sherrington, Rev. Mod. Phys. 42, 1 (1970).

[16] C.W. Gardiner, Quantum Noise (Springer, Berlin, 1991).

[17] D.F. Walls, Nature 306, 141 (1983); M. Teich and B.E.A. Saleh, Physics Today 43 (6), 26 (1990).

[18] M. Born and K. Huang, Dynamical Theory of Crystal Lattices (Oxford, Oxford, 1954).

[19] M. Schubert and B. Wilhelmi, Nonlinear Optics and Quantum Electronics (Wiley, New York, 1986).

[20] G.P. Srivastava, The Physics of Phonons (Hilger, Philadelphia, 1990).

[21] R. Loudon and P.L. Knight, J. Mod. Opt. 34, 709 (1987).

[22] X. Hu and F. Nori, unpublished.

[23] B.L. Schumaker, Phys. Rep. 135, 317 (1986). 
FIG. 1. Schematic diagram of the uncertainty areas (shaded) in the generalized coordinate and momentum $(X(\mathbf{q},-\mathbf{q})$, $P(\mathbf{q},-\mathbf{q}))$ phase space of (a) the phonon vacuum state, (b) a phonon number state, (c) a phonon coherent state, and (d) a phonon squeezed state. Here $X(\mathbf{q},-\mathbf{q})$ and $P(\mathbf{q},-\mathbf{q})$ are the two-mode $( \pm \mathbf{q})$ coordinate and momentum operators, which are the direct generalizations of their corresponding single-mode operators. Notice that the phonon coherent state has the same uncertainty area as the vacuum state, and that both areas are circular, while the squeezed state has an elliptical uncertainty area. Therefore, in the direction parallel to the $\theta / 2$ line, the squeezed state has a smaller noise than both the vacuum and coherent states.

FIG. 2. Schematic diagram of the time evolution of the expectation value and the fluctuation of the lattice amplitude operator $u( \pm \mathbf{q})$ in different states. Dashed lines represent $\langle u( \pm \mathbf{q})\rangle$, while the solid lines represent the envelopes $\langle u( \pm \mathbf{q})\rangle \pm \sqrt{\left\langle[\Delta u( \pm \mathbf{q})]^{2}\right\rangle}$. (a) The phonon vacuum state $|0\rangle$, where $\langle u( \pm \mathbf{q})\rangle=0$ and $\left\langle[\Delta u( \pm \mathbf{q})]^{2}\right\rangle=2$. (b) A phonon number state $\left|n_{\mathbf{q}}, n_{-\mathbf{q}}\right\rangle$, where $\langle u( \pm \mathbf{q})\rangle=0$ and $\left\langle[\Delta u( \pm \mathbf{q})]^{2}\right\rangle=2\left(n_{\mathbf{q}}+n_{-\mathbf{q}}\right)+2$. (c) A single-mode phonon coherent state $\left|\alpha_{\mathbf{q}}\right\rangle$, where $\langle u( \pm \mathbf{q})\rangle=2\left|\alpha_{\mathbf{q}}\right| \cos \omega_{\mathbf{q}} t$ (i.e., $\alpha_{\mathbf{q}}$ is real), and $\left\langle(\Delta u[ \pm \mathbf{q})]^{2}\right\rangle=2$. (d) A single-mode phonon squeezed state $\mid \alpha_{\mathbf{q}} e^{-i \omega_{\mathbf{q}} t}$, $\left.\xi(t)\right\rangle$, with the squeezing factor $\xi(t)=r e^{-2 i \omega_{\mathbf{q}} t}$ and $r=1$. Here, $\langle u( \pm \mathbf{q})\rangle=2\left|\alpha_{\mathbf{q}}\right| \cos \omega_{\mathbf{q}} t$, and $\left\langle[\Delta u( \pm \mathbf{q})]^{2}\right\rangle=2\left(e^{-2 r} \cos ^{2} \omega_{\mathbf{q}} t+e^{2 r} \sin ^{2} \omega_{\mathbf{q}} t\right)$. (e) A single-mode phonon squeezed state, as in (d); now the expectation value of $u$ is $\langle u( \pm \mathbf{q})\rangle=2\left|\alpha_{\mathbf{q}}\right| \sin \omega_{\mathbf{q}} t$, (i.e. $\alpha_{\mathbf{q}}$ is purely imaginary), and the fluctuation $\left\langle[\Delta u( \pm \mathbf{q})]^{2}\right\rangle$ has the same time-dependence as in (d). Notice that the squeezing effect now appears at the times when the lattice amplitude $\langle u( \pm \mathbf{q})\rangle$ reaches its maxima, while in $(\mathrm{d})$ the squeezing effect is present at the times when $\langle u( \pm \mathbf{q})\rangle$ is close to zero. 


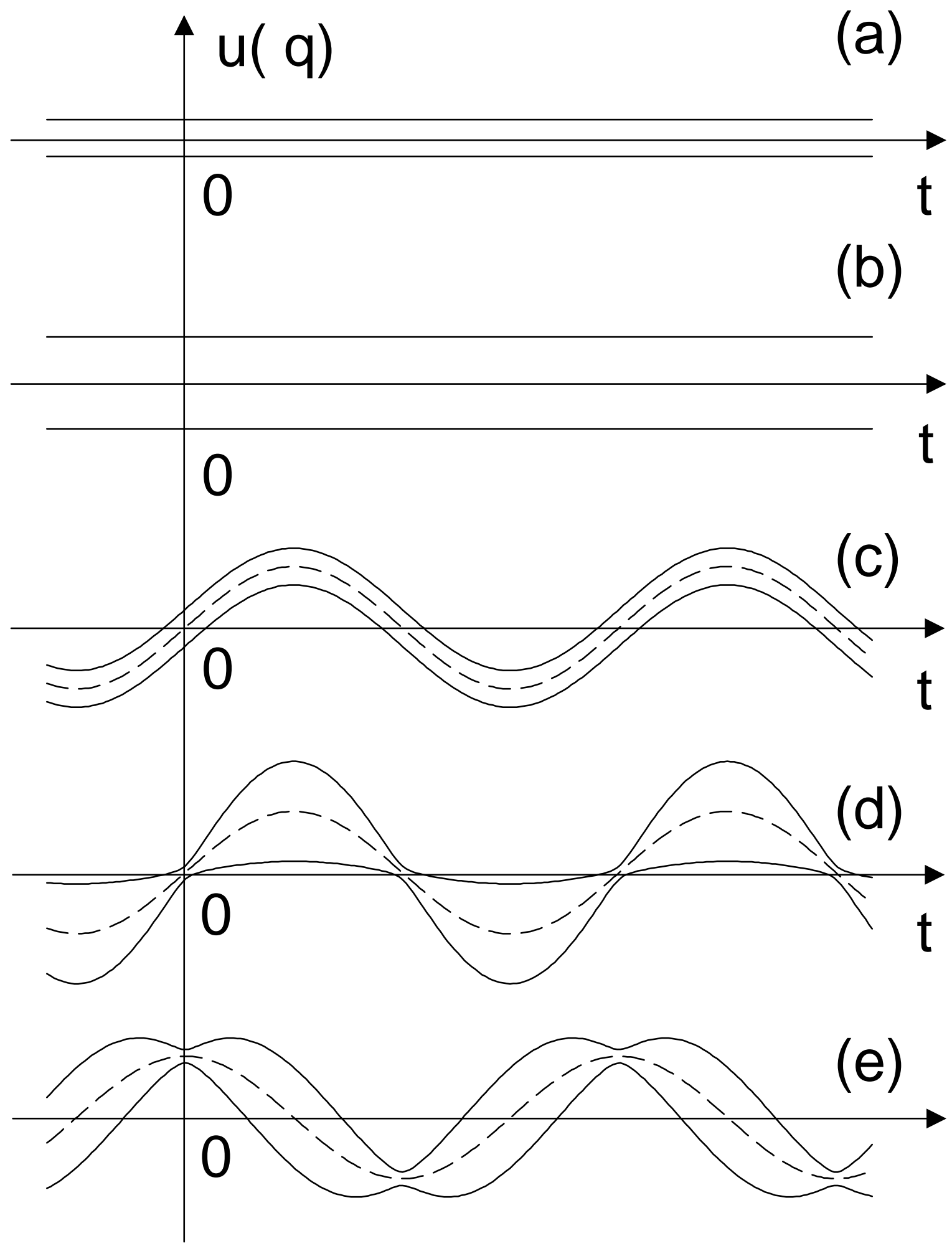


